\title{
Arbor
}

\section{La Educación en la estructura autonómica del Estado Español}

\section{Sofía Juaristi Zalduendo}

Arbor CLXXIII, 681 (Septiembre 2002), 55-65 pp.

A pesar del consenso logrado en materia educativa durante la transición política española con motivo de la aprobación de la Constitución, aún existen divergencias notables entre los partidos politicos sobre el modelo educativo, como se ha podido comprobar en el debate de la Reforma de las Ciencias, la Lengua y las Humanidades. Así, aparecen diferencias significativas entre los curricula de las diferentes Comunidades Autónomas que debieran desparecer con el fin de que los alumnos y alumnas no las sufran en sus traslados de una Comunidad a otra. En este sentido, resulta de suma importancia que el profesorado de cada una de ellas participe en su elaboración, pues de nada sirve la función legislativa si el estamento docente no asume como propias las reformas que se han de aplicar en las aulas. Finalmente, aún valorando positivamente la función que tuvo la L.R.U., se constata que la L.O.U. introduce un sistema adecuado para la evaluación y la acreditación de la calidad, articula los distintos niveles competenciales de las Universidades y las administraciones, diseña un mayor autogobierno de las Universidades y permite impulsar la movilidad de estudiantes, profesores e investigadores tanto dentro de la estructura autonómica española como en la Unión Europea.

Quizás, en estos nuevos tiempos en los que vive el Estado Español, los intereses «ideológicos» de los agentes sociales con implantación tanto na- 
cional como autonómica, están provocando demasiada información mediática que difumina el trabajo diario, la reflexión, la organización, la investigación y la formación permanente que la educación requiere. Esto sucede, especialmente, en unos momentos en los que, en la sociedad del conocimiento, los cambios de las diversas sociedades se amalgaman y acrisolan en una inmensa aldea global donde las diferentes culturas se influencian las unas de las otras, aunque existan evidentes anacronismos entre las mismas.

Raro es el día en que los Medios de Comunicación no abordan un tema educativo ya sea sobre la Ley de Universidades, la Ley de Calidad o la Ley de Formación Profesional, la política de conciertos, la homologación salarial, la convivencia escolar, y mil y un temas de suma importancia que nos llevan a reflexionar sobre ¿qué educación es la más conveniente para los jóvenes del Siglo XXI?

Las viejas teorías parecen desmoronarse ante los acontecimientos de los últimos tiempos. El modelo familiar evoluciona según lo requiere la sociedad, y las fórmulas de convivencia y de relación interpersonal son múltiples, provocando diversos modos individuales de pensar y de sentir, tan heterogéneas y, en ocasiones, tan discordantes, que obligan al profesorado a adoptar posiciones eclécticas en las aulas en lo concerniente a la multiplicidad de valores. Por todo ello, en muchos casos, el alumnado no encuentra los referentes que necesita ni en la familia, ni en la escuela, por lo que debe buscar sus modelos en los medios de comunicación y en la calle, modelos que suelen coincidir con sus mitos favoritos y con los de su pandilla.

En la actualidad, tras la puesta en práctica de la L.O.G.S.E., muchas son las voces que se alzan a favor, en contra y por la reforma de la misma, publicándose recientemente dos Reales Decretos ${ }^{1}$ por los que se establecen las enseñanzas mínimas de la educación Secundaria Obligatoria y del Bachillerato, dentro de un gran debate sobre la necesidad de potenciar y reforzar las Ciencias, la Lengua y las Humanidades.

Lo cierto es rue en el Estado Español aún continúan existiendo muchos ciudadanos que añoran una educación que, a la manera de la que presumía la Institución Libre de Enseñanza, sea completamente ajena a todo interés de comunión religiosa, escuela filosófica o partido político. De acuerdo con esta posición, se proclamaría tan solo el principio de libertad e inviolabilidad de la ciencia y de la consiguiente independencia de su indagación y exposición respecto de cualquier otra autoridad que no sea la de la propia conciencia del profesor, único responsable de su doctrina. Pero también existen otros ciudadanos que consideran que la educación ha de basarse en una escuela filosófica o comunión religiosa 


\section{La Educación en la estructura autonómica}

que inspire su ideario, con el fin de que los padres puedan elegir el centro que desean de acuerdo con los valores en los que quieren que se eduquen sus hijos, lo que evidencia un mapa heterogéneo que se repite en todas las Comunidades Autónomas, donde algunos profesores de la Escuela Pública y de las Cooperativas, especialmente, continúan perfeccionando la ya vieja escuela de la denominada «nueva educación» de María Montessori ${ }^{2}$, Ovide Decroly ${ }^{3}$ y Freinet ${ }^{4}$, adecuando los métodos a los tiempos actuales con la utilización de las tecnologías.

Sin embargo, este no es el tema principal de debate, aunque en estos momentos sería de enorme interés, porque se plasmarían de forma evidente las razones pedagógicas y didácticas de los métodos más adecuados para una educación innovadora del nuevo siglo. El debate suele estar más centrado en conseguir que los Partidos lleguen a consensuar y, si no es así, retrasar las Leyes de Universidades, Calidad y F.P., promovidas por grupos políticos (hoy el Partido Popular ) que cuenten con la mayoría absoluta.

No olvidemos que uno de los grandes éxitos de la transición política fue el consenso de la derecha y de la izquierda en torno a la Constitución; acuerdo básico sobre el punto de partida de nuestro ordenamiento jurídico que no se consiguió sin un gran esfuerzo político, especialmente, como afirma Manuel de Puelles Benítez (1991, pp. 484-485), «cuando hubo que regular derechos llenos de una gran carga ideológica. Así ocurrió con la educación, materia extraordinariamente controvertida que estuvo a punto de quebrar el deseado consenso». El artículo 27 fue fruto, pues, de una complicada arquitectura dialéctica, y de este modo el Partido Socialista aceptaba el derecho de los padres a que sus hijos recibieran formación religiosa y moral de acuerdo con sus propias convicciones (apartado tercero), resolviéndose el viejo pleito del laicismo escolar, si bien el Partido centrista aceptaba el principio de la voluntariedad de la religión en las escuelas y no su imposición. Además el Partido Socialista aceptaba no sólo la existencia de una red privada de centros docentes, sino también el que los poderes públicos asumiesen la obligación de ayudar a los centros privados siempre que reúnan los requisitos que la ley establezca (apartado noveno).

Razón tiene, pues, Puelles Benítez (1991, p. 485), al afirmar que «el artículo 27 no es más que el difícil equilibrio entre los principios de igualdad y libertad en educación. Mientras la izquierda hacía énfasis en el principio de igualdad -y por tanto en el derecho a la educación y en la participación e intervención de la comunidad escolar-, la derecha hacia hincapié en el principio de libertad centrado fundamentalmente en la creación de centros privados. En todo caso, se trataba de un pacto constitucional de difícil ejecución». 


\section{En torno a la organización territorial del Estado español}

La Constitución Española, sancionada por el Rey ante las Cortes el 27 de diciembre de 1978, fue publicada en el B.O.E el 29 de diciembre, y en el título VIII, «De la organización territorial del Estado», aborda una nueva configuración de España, e implanta las vulgarmente llamadas «vía rápida» y «vía lenta» para acceder a la autonomía en los artículos $151^{\circ}$ y $143^{\circ}$.

En la actualidad las transferencias en materia de Educación son una realidad en Andalucía, Aragón, Baleares, Canarias, Cantabria, Castilla y León, Castilla la Mancha, Cataluña, Comunidad Valenciana, Extremadura, Galicia, Madrid, Murcia, Navarra, País Vasco, Principado de Asturias y La Rioja. Consecuencialmente, el Ministerio de Educación, Cultura y Deporte, con más de cien años desde su creación como Ministerio de Instrucción Pública y Bellas Artes en 1900, ha visto reducido su ámbito de competencias plenas en materia educativa a Ceuta y Melilla, por lo que la heterogeneidad legislativa de las Comunidades Autónomas pueden potenciar una desvertebración del sistema educativo en el Estado Español.

No es cuestión de hacer una comparación normativa entre las diferentes Comunidades Autónomas desde que asumieron las transferencias en materia educativa, pero si preguntamos a los docentes de la Enseñanza Pública, enseguida se referirán a las diferencias salariales entre las distintas Autonomías, hecho peculiar, de muy difícil explicación, y en los distintos Cuerpos a los que se accede con idéntica titulación.

Por ello, es necesario destacar la fijación existente de las cuotas de participación de las diversas Comunidades en la elaboración de los $\mathrm{cu}$ rricula. De esta manera, el Ministerio fija los contenidos mínimos, comunes para todos los escolares españoles, cualquiera que sea el lugar en donde residan, suponiendo esos mínimos el $55 \%$ del curriculum en aquellas Comunidades Autónomas con lengua propia, y elevándose, en las demás al $65 \%$. El resto del curriculum, $45 \%$ en unos casos, $35 \%$ en otros, corresponde a las Autonomías.

Y he ahí la preocupación de los ciudadanos, pues de utilizarse la educación como materia de discordia política, en vez de intentar lograr amplios acuerdos, estables y duraderos entre todos los Gobiernos de las distintas Autonomías, se corre el peligro de que los alumnos de unas Comunidades Autónomas no adquieran niveles semejantes de conocimientos, procedimientos y destrezas en diversas materias. Puede llegar a producirse tal heterogeneidad en el \% del curriculum diseñado por las Autonomías, que pueda darse el hecho de que los conocimientos adquiridos 
en un nivel de la Enseñanza Obligatoria en una Comunidad Autónoma, necesariamente deban ser mayores, menores o diferentes que en otra Comunidad Autónoma dentro del mismo territorio español.

\section{El debate sobre la Reforma de las Humanidades en Educación Secundaria}

Donde más ha surgido la polémica, especialmente por el clamor de las Comunidades llamadas «históricas», ha sido con motivo de la Reforma de las Humanidades, hasta el punto que Esperanza Aguirre, primera Ministra de Educación del Partido Popular en la Democracia Española en el nuevo período constitucional, se vio obligada a retirar el proyecto de Decreto de Reforma de la Humanidades en la Educación Secundaria Obligatoria en el pleno del Congreso de los Diputados el 16 de diciembre de 1997. El tema lo retomará Pilar del Castillo, Ministra de Educación, Cultura y Deporte, tras la mayoría absoluta del Partido Popular en las Elecciones Generales del 2000, cuando pueda llevar al B.O.E. los Reales Decretos de «Enseñanzas Mínimas de la Enseñanza Secundaria Obligatoria y del Bachillerato».

Jon Juaristi, Director del Instituto Cervantes, ha afirmado en el II Congreso de la Lengua celebrado en Valladolid que «debemos afrontar juntos los riesgos y las esperanzas de los tiempos nuevos, porque como pueblo no tendremos otro destino que el de nuestra lengua común» ${ }^{5}$; lengua que une y vincula a todas las Comunidades Autónomas del Estado, incluidas las que poseen su lengua propia. Porque como ha aseverado el escritor mexicano Carlos Fuentes en el mismo Congreso, «contra una globalización unificadora, prefiero el mestizaje (...) El español es una lengua impura y en su impureza reside su valor y su comunicabilidad (...) La maror parte de los que hablamos español no pertenecemos a una sola raza, no que somos descendientes de indígenas, negros, europeos y todos los istizajes de por medio. Y en Europa, España es acaso el país más mesuzo: celtíbero, fenicio, griego, romano, godo, judío y árabe» 6 .

Por ello, es difícil de explicar la polémica surgida (especialmente en las Comunidades Autónomas), hasta la culminación de la publicación en el B.O.E. de los Reales Decretos citados, cuando una vez alcanzado el objetivo de extender la Educación Obligatoria hasta los 16 años y tras diez años de aplicación de la L.O.G.S.E., estudios realizados por diferentes instituciones ponían de relieve un conjunto de disfunciones que aconsejaban una decidida revisión de los planes de estudio, encamina a asegurar la mejora de la calidad en la sociedad del conocimiento del siglo XXI. 


\section{Sofía Juaristi Zalduendo}

Sin embargo, seis meses antes de que la Ministra, Pilar del Castillo, resolviese, mediante la oportuna medida legislativa, una cuestión que en la legislatura anterior había provocado una sonada derrota parlamentaria del Gobierno y había agitado tanto a la opinión pública como a los medios de comunicación con la publicación de más de setecientos artículos sobre la materia, Javier Tusell afirmaba «el problema crucial no se plantea en este terreno general sino en relación con la pluralidad de España y la enseñanza de la Historia (...) De ahí que un mero calificativo -el propósito de explicar la Historia «unitaria»- levantara barricadas. Los historiadores, sin embargo, estamos mucho más de acuerdo de lo que pueda pensarse. Ninguno negará, por ejemplo, que existe una trayectoria común a lo largo de los siglos, pero tampoco que eso que llamamos España ha sido una sociedad más plural desde hace mucho tiempo que ninguna otra de Europa occidental. No hay una nación europea en que más del $40 \%$ de sus habitantes convivan con otras tres lenguas oficiales, aparte de la más difundida, y en que, al mismo tiempo, quienes tienen una cultura más diferenciada -vascos y catalanes- se sientan en su mayoría españoles» ${ }^{7}$.

Y en el mes de julio de 2000, en el Monasterio de Yuso, cuna del castellano, los portavoces en el Congreso y en el Senado del Partido Popular, con Javier Arenas, Secretario General del Partido Popular, al frente, hacen pública la declaración de San Millán de la Cogolla en favor de las Humanidades aseverando que «creemos necesario asegurar a los alumnos el dominio oral y escrito de la lengua castellana y, en su caso, de la lengua oficial propia de la Comunidad Autónoma, así como un suficiente conocimiento de la literatura de las lenguas españolas y de la literatura universal; familiarizar a nuestros estudiantes en el uso de bibliotecas y otras fuentes de información, con la utilización de las nuevas tecnologías; reforzar el estudio de la historia, con respeto a los hechos históricos mismos y con la necesaria dimensión cronológica, que ha de incluir el estudio del pasado común de España y abrirse a una visión universal; dar a conocer la realidad plural de España y de Europa, y facilitar el estudio de sus lenguas; potenciar la enseñanza de la filosofía, sin prescindir del enfoque histórico; asegurar la presencia efectiva de la cultura clásica, así como del latín y del griego en el Bachillerato; promover la enseñanza de la historia del arte, de la formación musical y de la danza; mejorar las enseñanzas de contenido científico y tecnológico. Todo ello con un debido apoyo al profesorado y a su dignidad profesional».

A principios de ese mes de julio de 2000 los principales medios de Comunicación nacionales se habían hecho eco de la extraordinaria polémica política desatada por el informe de la Real Academia de la Historia 


\section{La Educación en la estructura autonómica}

sobre el contenido de los libros de texto de esta materia dirigidos a los alumnos de la E.S.O. y el Bachillerato, en el que se afirma que «en el proyecto de reforma de los años ochenta, se insistía en la necesidad de asegurar las bases pedagógicas de la actividad docente. Esta idea, en principio loable, tuvo, no obstante, efectos negativos en lo que se refiere al ámbito educativo. La tradicional enseñanza de la historia del bachillerato, se repetía por doquier, se había caracterizado por estar ayuna de elementos didácticos. Era la «historia sin pedagogía», como se la ha calificado recientemente. Pero la conveniencia que en absoluto negamos, de que el profesor de enseñanza secundaria conozca los fundamentos de la psicopedagogía fracasó en lo que se refiere a la enseñanza de la historia. Partiendo de las ideas que se recogían en el folleto «Los presupuestos didácticos de la reforma», en los que podía leerse que "se trata de ir más allá de las enseñanzas académicas tradicionales y de atender a otros aspectos que suponen desarrollo de capacidades, de hábitos, de actitudes», se criticaba la denominada "historia académica». Se la consideraba centrada en la cronología y en los acontecimientos. Se pensaba que lo importante no era saber más o menos cosas del pasado, sino adquirir las destrezas propias de la disciplina, lo que se podía conseguir tanto si se estudiaba la revolución francesa como la historia de la iglesia contigua al centro de enseñanza de que se tratara. Ante tales planteamientos, se podía objetar que «no es posible conseguir una visión general del pasado mediante una disciplina convertida en historia local, discontinua y particularista» 8 .

En la Introducción al libro de Julio Rodríguez Frutos (1989, p. 10), ya se recogían estas opiniones, llegando a asegurar que «las nuevas visiones de evaluación difícil y definitiva parecen estar cargadas, igualmente, de innumerables lastres: la enseñanza activa se convierte en activismo desenfrenado; el abuso de los medios, técnicas y métodos, carentes de cualquier investigación, no sólo no entretienen a los alumnos sino que se convierten en desconcierto y frustración para muchos profesores y alumnos que, al final, retornan a los viejos métodos; se merman los contenidos desvirtuando el conocimiento histórico hasta hacerlo irreconocible; se lleva la enseñanza de la historia local hasta lo grotesco».

\section{Reformas legales y realidad escolar}

Pensar que el debate pedagógico y didáctico sobre el que se sostenía el debate político en el área de las Humanidades, como en otras áreas, se ha disipado, sería de una ingenuidad casi irresponsable. De nada sirve 
publicar en el Boletín Oficial del Estado dos Decretos estableciendo las enseñanzas mínimas de la Educación Secundaria Obligatoria y el Bachillerato, si la metodología a aplicar no es asumida por el profesorado que, al final, será quien abordará día a día los contenidos de acuerdo con su criterio, y potenciará de manera subjetiva las destrezas y los hábitos; de ahí que sea tan importante en la aplicación de cualquier reforma el contar con la opinión mayoritaria de los docentes que, al final, van a ser los que van a llevar a cabo el currículo aprobado.

Por ello, las Comunidades Autónomas, que tienen la responsabilidad de desarrollar el diseño curricular de las mismas, en su tanto por ciento correspondiente, es lógico que el diseño que elaboren sea entregado al mayor abanico de agentes sociales, a los miembros de la Comunidad Educativa, y dentro de ésta, principalmente al profesorado, con el fin de que no vuelva a reproducirse en cada Comunidad Autónoma la dialéctica surgida anteriormente a nivel estatal con la reforma de las Humanidades.

Sin embargo, siendo este proceso vital para la búsqueda de un diseño curricular propio que cuente con el mayor consenso posible, no es menos cierto que las Comunidades Autónomas deben igualmente consensuar uno que se articule bajo los mismos parámetros, y cuyos contenidos no difieran de tal manera que parezcan sistemas educativos diferentes.

Que no se haga realidad la frase de Francisco Javier Jordán, profesor de psicopedagogía, de «enseño, luego sobrevivo» ${ }^{9}$, en unos momentos donde la participación democrática es una realidad y en los que todos los días pueden deparar nuevos descubrimientos, que nos permitan mejorar aún más la calidad de la enseñanza teniendo la excelencia como objetivo, sin olvidar que únicamente una escuela vanguardista con visión de futuro es capaz de desarrollar una educación previsora que, anticipándose al nuevo devenir, consigan que los Centros de Enseñanza continúen siendo los faros del Saber, de la Ciencia y de la Convivencia; y más, en unos momentos donde los medios de Comunicación no buscan la excelencia en su programación, sino que, cogiendo el tajo más corto, renuncian en demasiadas ocasiones a la calidad, en su desaforada búsqueda por la máxima audiencia, sin detenerse a pensar en el deterioro social que provocan, y por el que acaban resintiéndose la familia y la escuela.

\section{Concepto de Universidad y estructura autonómica del Estado}

El derecho fundamental de la autonomía de las Universidades que reconoce la Constitución española tuvo en la ley orgánica de Reforma Uni- 
versitaria (L.R.U.) 11/1983, de 25 de agosto, su norma de desarrollo que se ha visto completada por los Estatutos de las Universidades.

Las características principales de dicha Ley pueden fijarse en la consideración de la Universidad como institución educativa prestadora de un servicio público; la consideración de la Universidad como un sistema autónomo en su conjunto; la consideración de los departamentos, no de la cátedra, como la base del sistema universitario. Y con su aprobación las Universidades se dieron a sí mismas sus estatutos o normas de gobierno; elaboraron sus propios planes de estudios de acuerdo con las directrices que, emanadas del Consejo de Universidades, aprueba el Gobierno; expidieron los títulos académicos; elaboraron sus propios presupuestos y adquirieron una autonomía de personal por el que pudieron seleccionar tanto al personal administrativo como al docente, pero especialmente la L.R.U. tuvo también otros fines que es necesario destacar, como son la delimitación competencial entre el Estado, las comunidades autónomas y las propias Universidades, y, sobre todo, la aproximación de la Universidad a la sociedad mediante la creación del Consejo Social

Todos somos conscientes que la nueva sociedad del conocimiento en la que nos encontramos exige una Universidad moderna y activa, capaz de renovarse constantemente con el fin de estar permanentemente en perfecta sintonía con las nuevas vías de progreso y, a la vez, ser garantía de conservación de un acervo científico y humanístico sin el cual, la sociedad perdería el verdadero sentido de su continua evolución, que no debe ser otro que el de una justicia social que sea capaz de mantener la paz y la prosperidad de los pueblos.

La Universidad Española, las diversas Universidades del Estado Español, han sufrido progresivos cambios en poco tiempo, y donde la L.R.U. abría nuevas esperanzas e ilusiones al final del pasado Siglo XX, a principios del Siglo XXI y del III Milenio se convertía en un infranqueable muro que impedía la renovación cotidiana capaz de mantener el espíritu universitario de acuerdo con los tiempos, que justifica su derogación y la aprobación por las Cortes Generales de la Ley Orgánica de Universidades 6/2001, de 21 de diciembre, ya que la reforma era necesaria y existía una base de consenso suficiente como para afirmar que responde a una exigencia de la sociedad española mayoritariamente sentida.

Si hacemos una revisión de la historia, los que hemos vivido la evolución de la Universidad en los últimos años 20, 25 o 30 años, entendíamos que era necesario una serie de cambios y de reformas concretas. Pero, sobre todo y muy fundamentalmente, entendíamos que había que introdu- 
cir procedimientos que facilitasen la eficacia y la agilidad de la gestión, y que fomentasen el desarrollo de la actividad investigadora. Concretamente, respecto de esta actividad, la Universidad de Cantabria se encuentra en la mejor posición dentro del conjunto de las 60 Universidades públicas. Aquí entendíamos que era necesario insertar mecanismos de colaboración de las empresas y de la sociedad, que proporcionasen a la Universidad algunos medios de financiación para continuar impulsando esta actividad.

Para un Gobierno que busca la gestión equilibrada de una Comunidad el problema de los recursos es una causa de seria reflexión, no solamente política, sino también moral. El problema de la gestión de los recursos que llegan a la Universidad es una preocupación constante dentro de la autonomía y las responsabilidades de la Comunidad Autónoma, y la L.O.U. introduce fundamentalmente un sistema adecuado para la evaluación y la acreditación de la calidad, objetivo prioritario de reconocimiento de la actividad de tantas personas que trabajan dentro de la Universidad a las cuales la sociedad debe pedirles unos resultados concretos.

No olvidemos que la L.O.U. articula los distintos niveles competenciales, los de las Universidades, las Comunidades Autónomas y la Administración General del Estado; a la vez que diseña un mayor autogobierno de las Universidades y supone un incremento del compromiso de las Comunidades Autónomas, lo que implica para las primeras una mayor eficiencia en el uso de los recursos públicos y nuevas atribuciones de coordinación y gestión para las segundas. Esto implica dotar de nuevas competencias a las Universidades y a las Comunidades Autónomas respecto a la anterior legislación, con el objetivo de plasmar en el texto de forma inequívoca la confianza de la sociedad en sus Universidades y la responsabilidad de éstas ante sus respectivas Administraciones educativas.

Y, principalmente, la nueva Ley Orgánica de Universidades permite e impulsa la movilidad, tanto de estudiantes como de profesores e investigadores, dentro del sistema español, pero también del europeo e internacional, ya que las políticas de movilidad deben ser determinantes, especialmente en la España de las Autonomías, para que los estudiantes puedan escoger libremente los centros y titulaciones más adecuados a sus intereses personales y profesionales, elección real que tienen reconocida como un derecho y está a su alcance a través del distrito universitario abierto, que va a redundar sin duda alguna en la mejora global del sistema universitario en cada una de las distintas Universidades ubicadas en todo el territorio nacional dentro del nuevo marco internacional y europeo. 


\section{La Educación en la estructura autonómica}

\section{Notas}

1 Real Decreto 3473/2000, de 29 de diciembre, por el que se modifica el Real Decreto 1007/1991, de 14 de junio, por el que se establecen las enseñanzas mínimas correspondientes a la Educación Secundaria Obligatoria; y Real Decreto 3474/2000, de 29 de diciembre, por el que se modifican el Real Decreto 1700/1991, de 29 de noviembre, por el que se establece la estructura del Bachillerato, y el Real Decreto 1178/1992, de 2 de octubre, por el que se establecen las enseñanzas mínimas del Bachillerato.

2 El núcleo de la pedagogía montessoriana consiste en concebir esencialmente la educación como autoeducación, es decir, como un proceso espontáneo por medio del cual se desarrolla "el hombre que duerme ahí, y en considerar que, para que esto ocurra en el mejor de modos posibles, lo fundamental es proporcionar al niño un ambiente libre de obstáculos innaturales y materiales apropiados.

${ }^{3}$ Las bases del método Decroly son: respetar la aptitud del niño a apoderarse globalmente de los sectores de la experiencia que le suscitan un interés efectivo; organizar todas las actividades escolares en torno a "centros de interés" propios para cada edad; articular las actividades mismas en actividades de observación, de asociación y de expresión, con referencia en todos los casos a lo que constituye objeto actual de interés.

${ }^{4} \mathrm{La}$ técnica de la tipografía, si bien es esencial para el método Freinet, no adquiere significado sino en conexión con las otras «técnicas», a saber: 1) el texto libre; 2) la correspondencia interescolar; 3) el dibujo libre; 4) el cálculo viviente; 5) el libro de la vida; 6) ficheros y biblioteca de trabajo.

5 La Razón, 20 de octubre de 2001, p. 26.

${ }^{6}$ Ibidem, p. 26.

7 El Diario Montañés, 29 de junio de 2000, p. 21.

8 El País, 28 de junio de 2000.

9 Escuela Española, 4 de noviembre de 1999, p. 2.

\section{Bibliografía}

Abbagnano, N. y Visalberghi, A. (1978): Histaria de la Pedagogía. Madrid: Fondo de Cultura Económica.

MAGEE, B. (1999): Historia de la Filosofia. Barcelona: Art Blume S.L.

MAYORGa MANRIQUE, A. (2000): La Inspección Educativa. Siglo y Medio de la Inspección Educativa en España. 1849-1999. Madrid: Grupo Santillana de Ediciones, S.A.

M.E.C.D. (2001): Enseñanzas Minimas Educación Secundaria Obligatoria. Madrid: Secretaría General de Educación y Formación Profesional Ministerio de Educación, Cultura y Deporte.

M.E.C.D. (2001): Enseñanzas Mínimas Bachillerato. Madrid: Secretaría General de Educación y Formación Profesional, Ministerio de Educación, Cultura y Deporte.

Puelles Benftez, J. de (1991): Educación e ideología en la España Contemporánea. Barcelona: Editorial Labor S.A.

RODRfGUEZ Frutos, J. (1989): Enseñar Historia. Nuevas propuestas. Barcelona: Cuadernos de Pedagogía, Editorial Laia. 